\title{
Power Allocation and User Assignment Scheme for beyond 5G Heterogeneous Networks
}

\author{
Khush Bakht, ${ }^{1}$ Furqan Jameel, ${ }^{2}$ Zain Ali, ${ }^{3}$ Wali Ullah Khan $\mathbb{D}^{4},{ }^{4}$ Imran Khan $\left(\mathbb{D},{ }^{5}\right.$ \\ Guftaar Ahmad Sardar Sidhu, ${ }^{3}$ and Jeong Woo Lee ${ }^{6}{ }^{6}$ \\ ${ }^{1}$ Department of Electronic Engineering, Fatima Jinnah Women University, Rawalpindi 46000, Pakistan \\ ${ }^{2}$ Department of Communications and Networking, Aalto University, 02150 Espoo, Finland \\ ${ }^{3}$ Department of Electrical Engineering, COMSATS University, Islamabad 45550, Pakistan \\ ${ }^{4}$ School of Information Science and Engineering, Shandong University, Qingdao 266237, China \\ ${ }^{5}$ University of Engineering and Technology, Peshawar, Peshawar, Pakistan \\ ${ }^{6}$ School of Electrical and Electronics Engineering, Chung-Ang University, Seoul, Republic of Korea \\ Correspondence should be addressed to Jeong Woo Lee; jwlee2@cau.ac.kr
}

Received 8 June 2019; Revised 31 August 2019; Accepted 25 October 2019; Published 16 November 2019

Academic Editor: Olivier Berder

Copyright $\odot 2019$ Khush Bakht et al. This is an open access article distributed under the Creative Commons Attribution License, which permits unrestricted use, distribution, and reproduction in any medium, provided the original work is properly cited.

\begin{abstract}
The issue of spectrum scarcity in wireless networks is becoming prominent and critical with each passing year. Although several promising solutions have been proposed to provide a solution to spectrum scarcity, most of them have many associated tradeoffs. In this context, one of the emerging ideas relates to the utilization of cognitive radios (CR) for future heterogeneous networks (HetNets). This paper provides a marriage of two promising candidates (i.e., CR and HetNets) for beyond fifth generation (5G) wireless networks. More specifically, a joint power allocation and user assignment solution for the multiuser underlay CR-based HetNets has been proposed and evaluated. To counter the limiting factors in these networks, the individual power of transmitting nodes and interference temperature protection constraints of the primary networks have been considered. An efficient solution is designed from the dual decomposition approach, where the optimal user assignment is obtained for the optimized power allocation at each node. The simulation results validate the superiority of the proposed optimization scheme against conventional baseline techniques.
\end{abstract}

\section{Introduction}

In the last few years, the wireless systems have evolved to the point where homogeneous cellular networks have achieved near optimal performance [1]. These advancements in the homogeneous cellular networks, though significant, may not be enough to support beyond fifth generation (5G) wireless networks [2]. To do so, dynamic and exhaustive improvements in spectral efficiency are needed. One of the proposed solutions is advanced networks which consist of multiple tiers of high-powered base stations (BSs) overlaid with lowpowered BS both having different coverage areas, also known as heterogeneous networks (HetNets) [3]. Typically, a HetNet consists of main macro base stations (MBSs), a few pico base stations (PBSs), and several femto base stations
(FBSs). An MBS in HetNets has a high transmission power and greater coverage area which is then overlaid with lowpowered PBS and FBS $[4,5]$.

There are different purposes of PBS and FBS in the HetNets. Generally speaking, the PBS are laid in a dense traffic areas to improve coverage in hotspot areas, while FBS are overlaid in a manner to remove the coverage issues in homogeneous systems, thereby improving the overall performance. In conventional homogeneous cellular network, the mobile terminal is associated with a BS on the basis of downlink signal-to-interference-and-noise ratio (SINR). Specifically, a user is associated with a particular BS which offers SINR greater than the other BSs [6]. In HetNets, the SINR-based association principle leads to having load balancing issue among MBS and PBS. Hence, the network 
resources need to be intelligently distributed among BSs for higher spectral efficiency $[5,6]$.

1.1. Related Works. By the end of 2020, it is anticipated that up to 50 billion devices will exist in the world including static and mobile platforms [7]. Due to this reason, there has been an upsurge in the research on HetNets to provide efficient and long-term solutions [8]. Zhang et al. in [9] investigated the problem of user association in HetNets. They considered an optimization problem with different traffic capacity limits, quality of service (QoS) requirements, and power budget constraints. The proposed scheme of user association improved the performance of the network. A bit-rate adoption method was proposed by the authors of [10] for body area HetNets. Their scheme showed the promise of increasing the efficiency of packet transmission regardless of placement of the node. The authors of [11] performed joint optimization of resource allocation and user association in HetNets. They presented and compared three allocation strategies, i.e., orthogonal deployment, cochannel deployment, and partially shared deployment. Sequential quadratic programming- (SQP-)based power optimization approach was presented in [12] to maximize the sum rate of small-cell networks. Similar works have been done by the authors of [13], where a pricing-based approach for associating the user to a particular BS was proposed by the authors of [14], and the idea of a distributed price update strategy was presented for achieving the user fairness.

Liu et al. presented a fair user association scheme in [15]. They used the Nash bargaining solution (NBS), whereby the optimization with fairness was achieved among competing BSs. In [16], the HetNets using orthogonal frequency division multiple access (OFDMA) network were presented by the authors. The aim was to manage radio resource by maximizing the throughput of user having minimum rate. From the perspective of nonorthogonal multiple access (NOMA), the author of [17] provided a novel idea to use interference-aided vehicular networks and highlighted some key challenges. Similarly, the authors of [18] managed the radio resources by a scheduling algorithm implemented by a central global resource controller (GRC). The algorithm optimized the attributes such as fairness among users, spectral efficiency, and battery lifetime. Relay-based HetNets were considered by the authors of [19], wherein they proposed a method to suppress intercell and intracell interference. Their proposed scheme was shown to outperform existing baseline methods in terms of sum-rate performance. Another similar and much recent work [20] provided optimal time switching and power splitting technique for improving the performance of wireless network. The authors in [21] jointly optimized power allocation and user association in ultradense heterogeneous networks using noncooperative game theory, thus achieving the increase in system throughput as well as optimal power allocation.

Semov et al. proved that in HetNets by taking the geographical position into account, the user's throughput and fairness can be improved [22]. A similar concept was employed in the form of relays by the authors of [23] to ensure proportional fairness among user equipment by taking into account backhaul links between the relays and BS. On the other hand, the authors of [24] considered distributed antenna system (DAS) and compared cochannel resource allocation schemes for energy-efficient communication. In [25], the authors studied a single-cell HetNet having one macro and one picocell for efficient resource allocation such that the energy efficiency is maximized by proposing an iterative resource allocation algorithm. However, the same authors did not consider multicellular network for the evaluation. To achieve the spectral efficiency of the system more advanced dynamic spectrum access techniques (DSA) should be employed. The cognitive radio (CR) is an efficient DSA technique [26] that allows secondary (unlicensed) users (SU) to access the spectrum of primary (licensed) users (PU) in an opportunistic way [27]. In $\mathrm{CR}$, spectrum sharing can be classified as spectrum overlay and spectrum underlay. In spectrum overlay, the SU can transmit simultaneously on the frequency band used by the PU through adjusting its transmit power such that it does not cause much interference for the PU [27].

Of late, the CR-based HetNets have been drawing a lot of research attention nowadays due to their dynamic resource allocation property. The power adjustment of BS and mobile users can be achieved by dynamic resource allocation [28]. In this regard, the author in [29] maximized the energy efficiency subject to power and interference constraint in OFDMA-based CR networks. The authors applied the convex optimization theory and proposed an iterative algorithm. In [30], the authors considered a cognitive femtocell network using OFDMA and solved sum-utility maximization and dynamic resource allocation problem with the help of the dual decomposition method. In [31], the authors considered the stochastic optimization model for maximizing the long term energy efficiency in time-varying heterogeneous networks. The proposed problem is a mixed integer problem, and the Lagrange dual method has been used to solve it. In [32], the authors considered the resource allocation problem for rate maximization in multiuser cognitive heterogeneous networks. The authors considered the maximum transmit power of cognitive microcell base station and cross-tier interference constraints, simultaneously. They converted the nonconvex optimization problem into a geometric programming problem and solved it in a distributed way using the Lagrange dual method.

1.2. Motivation and Contributions. Although the research works reported in recent years have considered the overall system's performance maximization for CR-based HetNets, the problem of fairness among different users has received little attention. The potential problem arises when the schemes proposed for the sum-rate maximization assign very few or no resources to some of the users with higher fading conditions. This uneven distribution of resources results in degrading the achievable performance for different users. This problem may become more serious for the CRbased HetNets due to the limiting factor of increased interference. Thus, optimization of the transmission for user 
fairness under more practical constraints becomes essential. To the best of our knowledge, the resource optimization and user assignment techniques for fair rate allocation have not been jointly investigated in the literature due to the higher level of complexity involved in finding the optimal solution.

To fill this gap in the literature and provide a comprehensive solution to the user fairness problem in CR-based HetNets, we provide a joint strategy for power allocation and user fairness. In particular, we consider the joint power optimization and user association problem in HetNets for achieving fairness among different users. We first formulate a joint optimization problem subject to power and interference constraints. Then, to provide a less complex and an efficient solution, we design an algorithm from the dual decomposition strategy. The presented numerical results indicate the importance and utility of our scheme in comparison with the baseline methodologies.

1.3. Organization. The remainder of this paper is organized as follows. The system model and problem formulation are presented in Section 2. In Section 3, the proposed solution has been described, while Section 4 discusses the simulation results. Finally, Section 5 presents some concluding remarks and future research directions. In addition, the list of acronyms used throughout this paper has been provided in Table 1.

\section{System Model and Problem Formulation}

This section describes the considered system model and provides the steps related to problem formulation.

2.1. System Model. A downlink CR transmission is considered, where the secondary HetNet system is reusing the spectrum of the primary network in an underlay mode, as shown in Figure 1. The HetNet consists of a single macrocell overlaid with multiple pico BSs, intended to transmit data to multiple users. It is assumed that all the devices are equipped with a single antenna and experience independent and identically distributed (i.i.d) Rayleigh fading. The channel bandwidth is distributed among MBS and PBSs in such a way that MBS and PBSs are nonorthogonal to each other, while each PBS is orthogonal to other PBSs in the HetNet.

In this model, $\mathrm{BSs}$ are denoted as $\mathrm{BS}_{b}$, such that $\mathbb{B}=\mathrm{BS}_{b} \mid b=1,2,3, \ldots, B$, where $\mathrm{BS}_{1}$ is modeled as macro
TABLE 1: List of acronyms.

\begin{tabular}{lc}
\hline Acronym & Definition \\
\hline $5 G$ & Fifth generation \\
AWGN & Additive white Gaussian noise \\
BS & Base station \\
CR & Cognitive radio \\
D2D & Device to device \\
DAS & Distributed antenna system \\
DSA & Dynamic spectrum access \\
FBS & Femto base station \\
GRC & Global resource controller \\
HetNet & Heterogeneous networks \\
KKT & Karush-Kuhn-Tucker \\
MBS & Macro base station \\
NBS & Nash bargaining solution \\
OFDMA & Orthogonal frequency division multiple access \\
PBS & Pico base station \\
PSD & Power spectral density \\
PU & Primary user \\
QoS & Quality of service \\
RRM & Radio resource management \\
SQP & Sequential quadratic programming \\
SU & Secondary user \\
SINR & Signal-to-interference plus noise ratio \\
\hline
\end{tabular}

and the remaining BSs act as pico BSs. Further, there are $A$ users and $C$ channels allocated to BS. The signal-to-interference-and-noise ratio (SINR) of the ath user from the $b$ th BS at the $c$ th channel is given as in [11].

$$
\operatorname{SINR}_{a, c, b}=\frac{P_{a, c, b} g_{a, c, b}}{P_{a, c, b}^{\prime} f_{a, c, b}+\sigma_{a}^{2}},
$$

where $P_{a, c, b}$ is the transmit power for the ath user connected to the $b$ th BS at $c$ th channel, $\alpha_{a, c, b}$ denotes the association variable which belongs to set $\{0,1\}, \sigma_{a}^{2}$ is the power spectral density (PSD) of the noise, while $g_{a, c, b}$ and $f_{a, c, b}$ are the channel gains from intended BS to user and from interfering BS to the user, respectively. Furthermore, $P_{a, c, b}^{\prime}$ denotes the transmit power of interfering BS.

2.2. Problem Formulation. One of our key objectives in this article is fair maximization of the data rate of each user in the network. To facilitate mathematical analysis, we introduce a binary variable $\alpha_{a, c, b}$, such that

$$
\alpha_{a, c, b}=\left\{\begin{array}{l}
1, \text { when the } a \text { th user is associated with the } b \text { th BS through the } c \text { th channel, } \\
0, \text { otherwise. }
\end{array}\right.
$$

Based on the above expression, the channel allocation follows

$$
\sum_{c=1}^{C} \sum_{b=1}^{B} \alpha_{a, c, b}=1, \quad \forall a=1,2,3, \ldots, A .
$$

To protect PUs from the interference and to improve the performance of the system, resource allocation at BSs need to be performed such that

$$
\sum_{a=1}^{A} \sum_{c=1}^{C} \sum_{b=1}^{B} \alpha_{a, c, b} P_{a, c, b} h_{a, c, b} \leq I_{\mathrm{th}}
$$




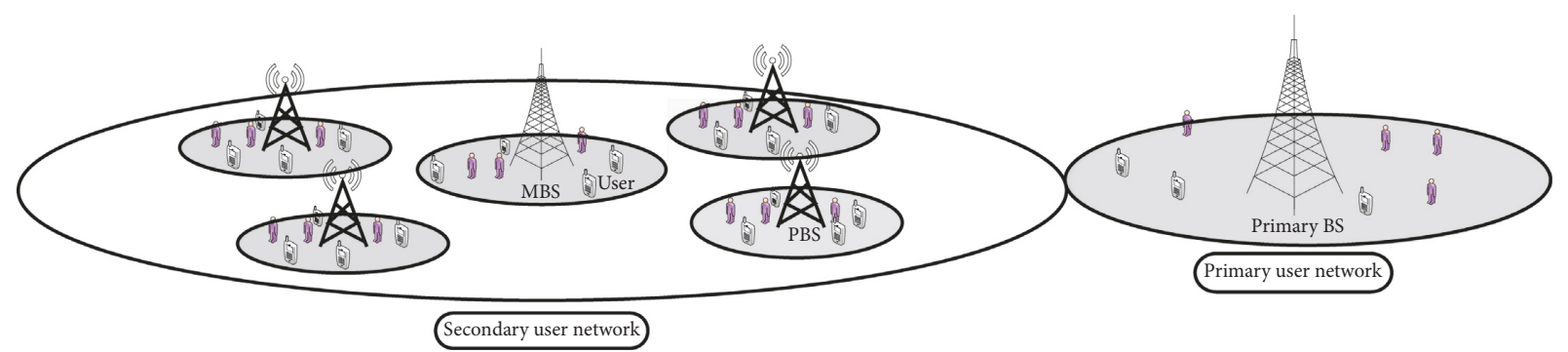

FIGURE 1: Heterogeneous network using cognitive radio spectrum in underlay mode.

where $P_{a, c, b}$ is the power allocated by the $b$ th BS to the $c$ th channel allocated to the ath user and $h_{a, c, b}$ represents the gain of interference channel $c$ from the $b$ th BS to primary BS. Finally, to ensure that the power consumed by each BS is less than or equal to the power budget, power allocation at each BS needs to be ensured as

$$
\sum_{a=1}^{A} \sum_{c=1}^{C} P_{a, c, b} \alpha_{a, c, b} \leq P_{b}, \quad \forall b=1,2,3, \ldots, B
$$

To achieve fairness in rates of different users, a max-minbased optimization framework is adopted such that the problem is stated mathematically as

$$
\begin{array}{cl}
\text { P1: } \max _{P_{a, c, b}, \alpha_{a, c, b}} & \min \log _{2}\left(1+\operatorname{SINR}_{a, c, b}\right), \\
\text { s.t. } & \text { (2), (3), and (4), }
\end{array}
$$

where $P_{b}$ denotes total power available at the $b$ th BS and $I_{\text {th }}$ represents the sum interference threshold. Here, the first constraint ensures that the total power allocated by the $b$ th BS must be within the available power budget. Similarly, the second constraint ensures that the primary system is well protected from interference due to the underlay communication, and the third constraint makes sure that the $c$ th channel is allocated to just one SU.

\section{Proposed Solution}

To provide a viable solution to the aforementioned problem, we propose a solution based on duality theory. As is evident from the above expression, the allocation of channels and power loading are strongly coupled variables, and thus a joint optimization approach is needed. The problem $\mathbf{P 1}$ is a mixed binary integer programming and requires an exhaustive search. Fortunately, it has been shown by [33] that for a sufficiently large number of subchannels, the gap between the dual solution and the primal solution reduce to zero, regardless of the nonconvexity of the original problem. Thus, we exploit the duality theory to decompose and optimally solve the coupled problem.

To convert the complex max-min problem into standard optimization form, we introduce intermediate variable $t$ such that

$$
t \leq \log _{2}\left(1+\operatorname{SINR}_{a, c, b}\right), \quad \forall a, c, b .
$$

The introduction of the intermediate variable transforms the problem as

$$
\begin{aligned}
\mathbf{P} 2: & \max _{P_{a, c, b}, t} t, \\
& \text { s.t. }
\end{aligned}
$$

To obtain an immediate solution of auxiliary variables, from the structure of objective in (8), we utilize the fact that for any $y \geq 0$, minimizing $y$ is equivalent to minimization of $y^{2}$. It is a known fact that maximizing $y$ is equal to minimizing $-y$. Hence, to transform the problem into a standard minimization problem, we replace the objective in (8) with its negative. After making these transformations P2 is written as

$$
\text { P3: } \min _{P_{a, c, b}, t}-t^{2}
$$

$$
\text { s.t. (2), (3), (4), and (6). }
$$

Now, substituting $x=-t$, we obtain

$$
\begin{aligned}
& \min _{P_{a, c, b}, t} x^{2} \\
& \text { s.t. (2), (3), (4), and }-x \leq \log _{2}\left(1+\operatorname{SINR}_{a, c, b}\right), \quad \forall a, c, b .
\end{aligned}
$$

The dual function associated with (10) is given by

$$
\begin{aligned}
& \begin{aligned}
D\left(\lambda_{a}, \eta_{b}, v\right)= & \min _{x, P_{a, c, b}, \alpha_{a, c, b}} \sum_{a=1}^{A} \lambda_{a}\left(-x-\sum_{c=1}^{C} \sum_{b=1}^{B} \alpha_{a, c, b} \log _{2}\left(1+\operatorname{SINR}_{a, c, b}\right)\right) \\
& +x^{2}+\sum_{b=1}^{B} \eta_{b}\left(\sum_{a=1}^{A} \sum_{c=1}^{C} \alpha_{a, c, b} P_{a, c, b}-P\right)+v\left(\sum_{a=1}^{A} \sum_{c=1}^{C} \sum_{b=1}^{B} \alpha_{a, c, b} P_{a, c, b} h_{a, c, b}-I_{\text {th }}\right)
\end{aligned} \\
& \text { s.t. } \sum_{c=1}^{C} \sum_{b=1}^{B} \alpha_{a, c, b}=1, \quad \forall a=1,2,3, \ldots A .
\end{aligned}
$$


The expression in (11) can be written as

$$
\begin{aligned}
D\left(\lambda_{a}, \eta_{b}, v\right)= & \min _{x, P_{a, c, b}, \alpha_{a, c, b}} x^{2}+\sum_{a=1}^{A} \sum_{c=1}^{C} \sum_{b=1}^{B} \alpha_{a, c, b}\left(-\lambda_{a} \log _{2}\left(1+S I N R_{a, c, b}\right)+\eta_{b} P a, c, b+v P_{a, c, b} h_{a, c, b}\right) \\
& -\sum_{a=1}^{A} \lambda_{a} x-\sum_{b=1}^{B} \eta_{b} P_{b}-v I_{\text {th }} \\
\text { s.t. } \sum_{c=1}^{C} \sum_{b=1}^{B} \alpha_{a, c, b}= & 1, \quad \forall a=1,2,3, \ldots, A .
\end{aligned}
$$

For any given channel allocation, dual decomposition guides to solve the following subproblems

$$
\begin{gathered}
\mathbf{P} 4: \min _{x}\left(x^{2}-x \sum_{a=1}^{A} \lambda_{a}\right), \\
\text { P5: } \min _{P_{a, c, b}}\left(-\lambda_{a} \log _{2}\left(1+\operatorname{SINR}_{a, c, b}\right)+\eta_{b} P_{a, c, b}+v P_{a, c, b} h_{a, c, b}\right) .
\end{gathered}
$$

Using the KKT conditions to find the solution of the problems given in (13) and (14), we get

$$
\begin{aligned}
x^{*} & =\frac{1}{2}\left(\sum_{a=1}^{A} \lambda_{a}\right)^{+}, \\
P_{a, c, b}^{*} & =\left(\frac{\Phi_{a, c, b}-P_{a, c, b}^{\prime} \alpha_{a, c, b} f_{a, c, b}}{g_{a, c, b}\left(\eta_{b}+v h_{a, c, b}\right)}\right)^{+},
\end{aligned}
$$

where $\Phi_{a, c, b}=\lambda_{a} g_{a, c, b}+\sigma_{a}^{2} \eta_{b}+v h_{a, c, b}$, for all $a, c, b$, when $(\Psi)^{+}=\max (0, \Psi)$. The detailed derivation steps have been provided in the Appendix.

Now, to find the optimum value of $\alpha_{a, c, b}$, the following optimization problem is considered:

$$
\begin{array}{ll}
\min _{\alpha_{a, c, b}} & \sum_{a=1}^{A} \sum_{c=1}^{C} \sum_{b=1}^{B} \alpha_{a, c, b}-\left(\lambda_{a} \log _{2}\left(1+\operatorname{SINR}_{a, c, b}\right)+\eta_{b} P_{a, c, b}^{*}\right. \\
+ & \left.v P_{a, c, b}^{*} h_{a, c, b}\right), \\
\text { s.t. } & \sum_{c=1}^{C} \sum_{b=1}^{B} \alpha_{a, c, b}=1, \quad \forall a=1,2,3, \ldots, A .
\end{array}
$$

Evidently, the optimal solution can be found, such that

$$
\alpha_{a, c, b}^{*}=\left\{\begin{array}{l}
\text { for } a=\arg \min _{c}\left(-\lambda_{a} \log _{2}\left(1+\operatorname{SINR}_{a, c, b}\right)+\eta_{b} P_{a, c, b}^{*}+v P_{a, c, b}^{*} h_{a, c, b}\right), \\
\text { otherwise. }
\end{array}\right.
$$

The dual problem is convex; hence, subgradient method can be adopted to find the solution. The dual variables are updated at each iteration as

$$
\begin{aligned}
& \lambda_{a}^{(\mathrm{itr})}=\left(\lambda_{a}^{(\mathrm{itr}-1)}+\delta^{(\mathrm{itr}-1)} \times\left(-\sum_{b=1}^{B} \sum_{c=1}^{C} \log _{2}\left(1+\operatorname{SINR}_{a, c, b}\right)-x\right)\right), \\
& \eta_{b}^{(\mathrm{itr})}=\left(\eta_{b}^{(\mathrm{itr}-1)}+\delta^{(\mathrm{itr}-1)}\left(\sum_{a=1}^{A} \sum_{c=1}^{C} P_{a, c, b}-P_{b}\right)\right), \\
& v^{(\mathrm{itr})}=\left(v^{(\mathrm{itr}-1)}+\delta^{(\mathrm{itr}-1)} \times\left(\sum_{a=1}^{A} \sum_{c=1}^{C} \sum_{b=1}^{B} P_{a, c, b} h_{a, c, b}-I_{\mathrm{th}}\right)\right),
\end{aligned}
$$

where $\delta$ is the step size. To obtain the joint optimization solution, power loading and channel allocation are updated in each iteration.

\section{Simulation Results}

In this section, we present the performance of the proposed scheme. The simulation environment for the proposed schemes is MATLAB. The noise PSD is $\sigma_{a}^{2}=0.1$. For simulation, we have considered the maximum number of users in the system which is $A=30$, the total channels available in the system which is $C=20$, and the total BS available in the system which is $B=5$. For the sake of the evaluation, we have compared our proposed optimal allocation and optimal power scheme (OAOP) with baseline schemes, i.e., fixed channel allocation and optimal power loading (FAOP) and fixed channel allocation and fixed power loading (FAFP).

In Figure 2, peak-to-average rate ratio (PR) has been plotted against different parameters. Different values of PR show fairness among the users. The smaller values of PR indicate a more fair scheme. Moreover, the effect of changing picocell power $\mathrm{PR}_{p}$ on $\mathrm{PR}$ has also been shown. 




Figure 2: Peak-to-average ratio with changing picocell power.

The picocell power has been varied from 0.25 to 2 . Considering $P_{p}=0.25$, the percentage gap between FAFP and FAOP is $49.74 \%$, between FAFP and OAOP is $22.59 \%$, and between FAOP and OAOP is $45.42 \%$. It can be seen that the value of PR decreases with an increase in the power. This indicates that high picocell power causes high interference which results in decreasing the PR. Moreover, a comparison of the three schemes clearly indicates that the OAOP scheme is the fairest among all. This is because optimization on channel allocation along with optimal power loading makes the problem more flexible compared with the cases when only power loading is optimized and if channel allocation and power loading are fixed. Also, for $P_{p}=0.5$, the percentage gap between FAFP and FAOP is $49.83 \%$, between FAFP and OAOP is $25.44 \%$, and between FAOP and OAOP is $51.05 \%$. Finally, increasing the value to $P_{p}=2$, the percentage gap between FAFP and FAOP is $56.99 \%$, between FAFP and OAOP is $33.12 \%$, and between FAOP and OAOP is $58.11 \%$.

Figure 3 shows the $\mathrm{PR}$ with changing interference constraint $\mathrm{PR}_{I_{\mathrm{t}}}$. The interference constraint has been varied from 20 to 40 . Considering $I_{\text {th }}=20$, the percentage gap between FAFP and FAOP is $55.08 \%$, between FAFP and OAOP is $31.83 \%$, and between FAOP and OAOP is $57.79 \%$. Also, for $I_{\text {th }}=25$, the percentage gap between FAFP and FAOP is $53.39 \%$, between FAFP and OAOP is $30.57 \%$, and between FAOP and OAOP is $57.27 \%$. Moreover, if $I_{\text {th }}=30$, then the percentage gap between FAFP and FAOP is $52.41 \%$, between FAFP and OAOP is $29.95 \%$, and between FAOP and OAOP is $57.14 \%$. Further increasing the value to $I_{\text {th }}=35$, the percentage gap between FAFP and FAOP is $52.00 \%$, between FAFP and OAOP is $29.62 \%$, and between FAOP and OAOP is $57.11 \%$. Finally, increasing the value to $I_{\text {th }}=40$, the percentage gap between FAFP and FAOP is $51.86 \%$, between FAFP and OAOP is $28.91 \%$, and between FAOP and OAOP is $56.31 \%$. Comparison of schemes in terms of the percentage gap shows that as the interference constraint $I_{\text {th }}$ increases, the percentage gap is decreasing. Moreover, the average gap between the graphs of FAFP with FAOP and FAOP with OAOP scheme is near to each other. Graphs also show that OAOP outperforms all other schemes in terms of fairness. The reason behind this is the additional flexibility provided by optimum channel allocation.

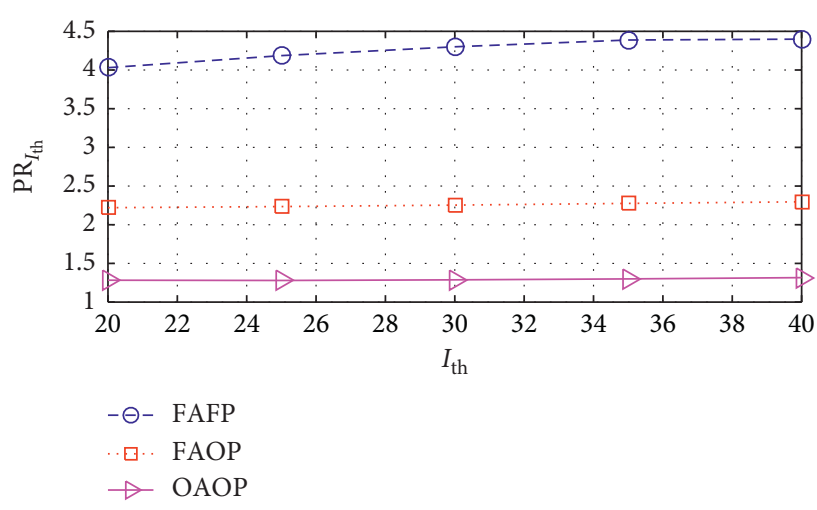

Figure 3: Peak-to-average ratio with changing $I_{\text {th }}$.

The effect of changing the power of macro base station $\mathrm{PR}_{m}$ on the peak-to-average rate ratio is shown in Figure 4. The macro power has been varied from 10 to 30 . Considering $\mathrm{PR}_{m}=10$, the percentage gap between FAFP and FAOP is $54.64 \%$, between FAFP and OAOP is $31.83 \%$, and between FAOP and OAOP is $58.26 \%$. Also, for $\mathrm{PR}_{m}=15$, the percentage gap between FAFP and FAOP is $56.05 \%$, between FAFP and OAOP is $34.46 \%$, and between FAOP and OAOP is $61.48 \%$. Moreover, if $\mathrm{PR}_{m}=20$, then the percentage gap between FAFP and FAOP is $56.65 \%$, between FAFP and OAOP is $36.27 \%$ and between FAOP and OAOP is $64.03 \%$. Further increasing the value to $\mathrm{PR}_{m}=25$, the percentage gap between FAFP and FAOP is $57.09 \%$, between FAFP and OAOP is $37.59 \%$, and between FAOP and OAOP is $65.84 \%$. Finally, increasing the value to $\mathrm{PR}_{m}=30$, the percentage gap between FAFP and FAOP is $57.49 \%$, between FAFP and OAOP is $38.61 \%$, and between FAOP and OAOP is $67.15 \%$. Hence, comparison of all the three schemes shows that the OAOP is the superior scheme.

In the results presented here, the sum throughput has also been maximized along with achieving fairness among the users. Figure 5 shows the sum throughput versus pico power $P_{p}$. The $P_{p}$ has been varied from 0.25 to 2 . The percentage gap between OAOP scheme and FAOP is 38\%, between OAOP and FAFP is 7\%, and between FAOP and FAFP is $18.42 \%$, where the value of $P_{p}=0.25$. The results evidently show that the spectral efficiency of OAOP schemes is the most optimal among the other power allocation schemes. This is because the channels are allocated to users in such a way that would maximize the total data rate of users. Then, increasing the value of $P_{p}=0.5$ the percentage gap between OAOP and FAOP is $42.56 \%$, between OAOP and FAFP is $7.825 \%$, and between FAOP and FAFP is $18.38 \%$. Further increasing the value to $P_{p}=2$, the percentage gap between OAOP and FAOP is $48.51 \%$, between OAOP and FAOP is $10.12 \%$, and between FAOP and FAFP is $20.85 \%$.

Figure 6 shows the sum throughput versus $I_{\text {th }}$. The percentage gap between OAOP scheme and FAOP is $45.98 \%$, between OAOP and FAFP is $9.73 \%$, and between FAOP and FAFP is $21.17 \%$, where the value of $I_{\text {th }}=20$. Further increasing the value to $I_{\text {th }}=25$ the percentage gap between OAOP and FAOP is $46.09 \%$, between OAOP and 


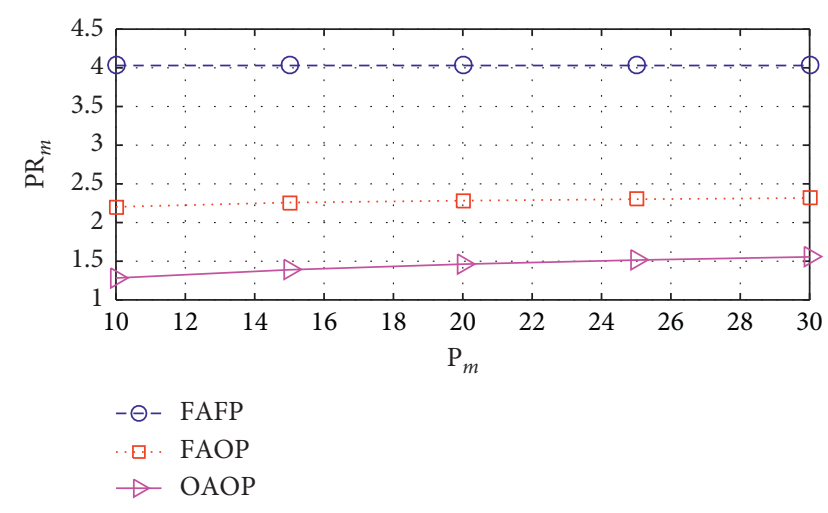

FIgURE 4: Peak-to-average ratio with changing macro power.

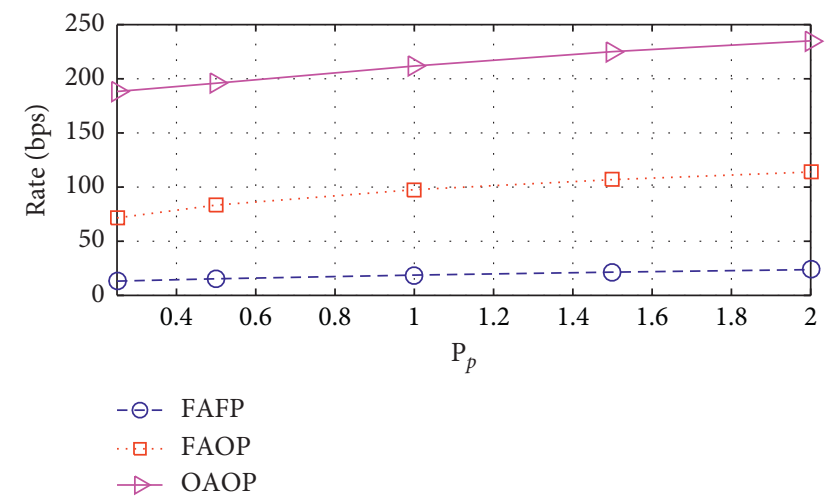

FIGURE 5: Sum throughput with changing pico power.

FAFP is $10.22 \%$, and between FAOP and FAFP is $22.17 \%$. Moreover, if $I_{\text {th }}=30$, percentage gap between OAOP and FAOP is $46.21 \%$, between OAOP and FAFP is $10.62 \%$, and between FAOP and FAFP is $23 \%$. Considering $I_{\text {th }}=35$, the percentage gap between OAOP and FAOP is $46.32 \%$, between OAOP and FAFP is $10.98 \%$, and between FAOP and FAFP is $23.71 \%$. Further increasing the value to $I_{\text {th }}=40$, the percentage gap between OAOP and FAOP is $46.47 \%$, between OAOP and FAOP is $11.01 \%$, and between FAOP and FAFP is $23.71 \%$. Hence, the sum throughput of OAOP scheme is maximum.

The effect of varying macro power $P_{m}$ on the sum throughput is shown in Figure 7 . The $P_{m}$ has been varied from 10 to 30 . The percentage gap between OAOP scheme and FAOP is $45.49 \%$, between OAOP and FAFP is $9.73 \%$, and between FAOP and FAFP is $21.40 \%$, where the value of $P_{m}=10$. Further increasing the value to $P_{m}=15$, the percentage gap between OAOP and FAOP is $46.69 \%$, between OAOP and FAFP is $9.65 \%$, and between FAOP and FAFP is $20.67 \%$. Moreover, if $P_{m}=20$, the percentage gap between OAOP and FAOP is $47.27 \%$, between OAOP and FAFP is $9.61 \%$, and between FAOP and FAFP is $20.32 \%$. Considering $P_{m}=25$, the percentage gap between OAOP and FAOP is $47.74 \%$, between OAOP and FAFP is $9.58 \%$, and between FAOP and FAFP is $20.06 \%$. Further increasing the value to $P_{m}=30$ the percentage gap between OAOP and FAOP is $48.13 \%$, between OAOP and FAOP is $9.56 \%$, and between FAOP and FAFP is $19.86 \%$. The results show that OAOP scheme is the most optimal.



FIGURE 6: Sum throughput with changing $I_{\text {th }}$.

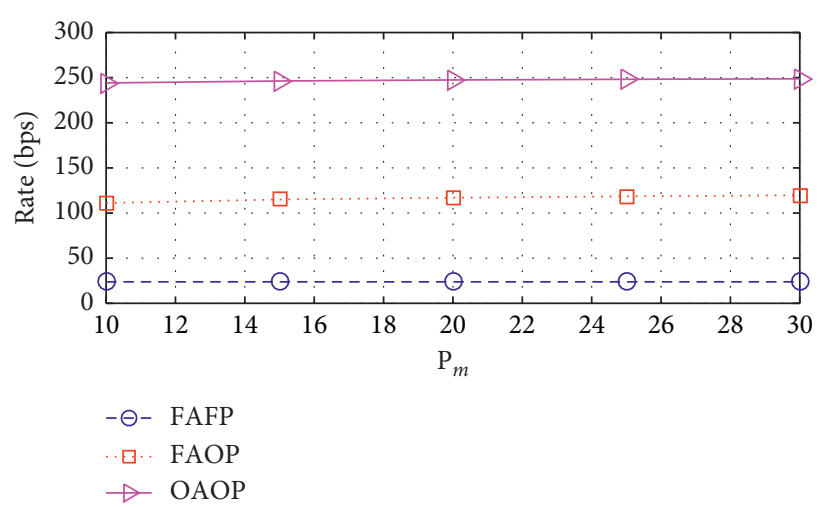

FIGURE 7: Sum throughput with changing macro power.

Figure 8 shows the values of sum throughput with a changing number of users $U$. From this plot, we identified that the performance of OAOP is far superior to other schemes which is due to the fact that adding new users to the system introduces new channel gains. This results in increasing the flexibility of the problem. In OAOP, both channel allocation and power loading are optimized; therefore, this scheme is better at taking advantage of the newly added users in the network. In FAOP, only power loading is optimized; so, the addition of new users increases the data rate because of the reduced degree of freedom compared with OAOP. A very slight increase is observed in FAFP when new users are added, as neither channel allocation nor power loading is optimized in this scheme. $U$ has been varied from 20 to 100 . Considering $U=20$, the percentage gap between OAOP scheme and FAOP is $61.07 \%$, between OAOP and FAFP is $14.82 \%$, and between FAOP and FAFP is $24.27 \%$. Further increasing $U=40$, the percentage gap between OAOP and FAOP is $53.28 \%$, between OAOP and FAFP is $10.99 \%$, and between FAOP and FAFP is $20.63 \%$. Moreover, if $U=60$, the percentage gap between OAOP and FAOP is $48.81 \%$, between OAOP and FAFP is $10.79 \%$, and between FAOP and FAFP is $20.10 \%$.

Figure 9 shows the convergence of dual variables $\lambda_{a}, \eta$, and $v$ with initial value as $0.6, \eta$ as 0.6 , and $v$ as 0.1 . The smaller the value of step size, the finer the convergence is, whereas a bigger value leads to fast convergence. 




FIgURE 8: Sum throughput with increasing users.

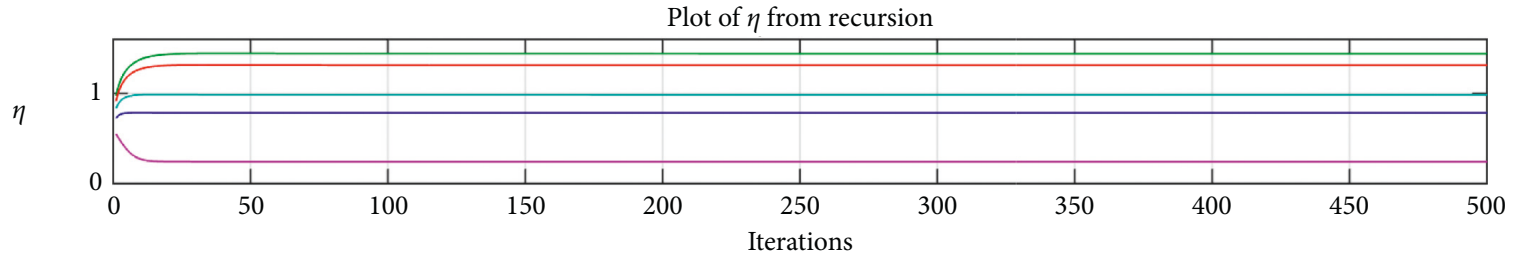

Plot of $\lambda$ from recursion

$\lambda$
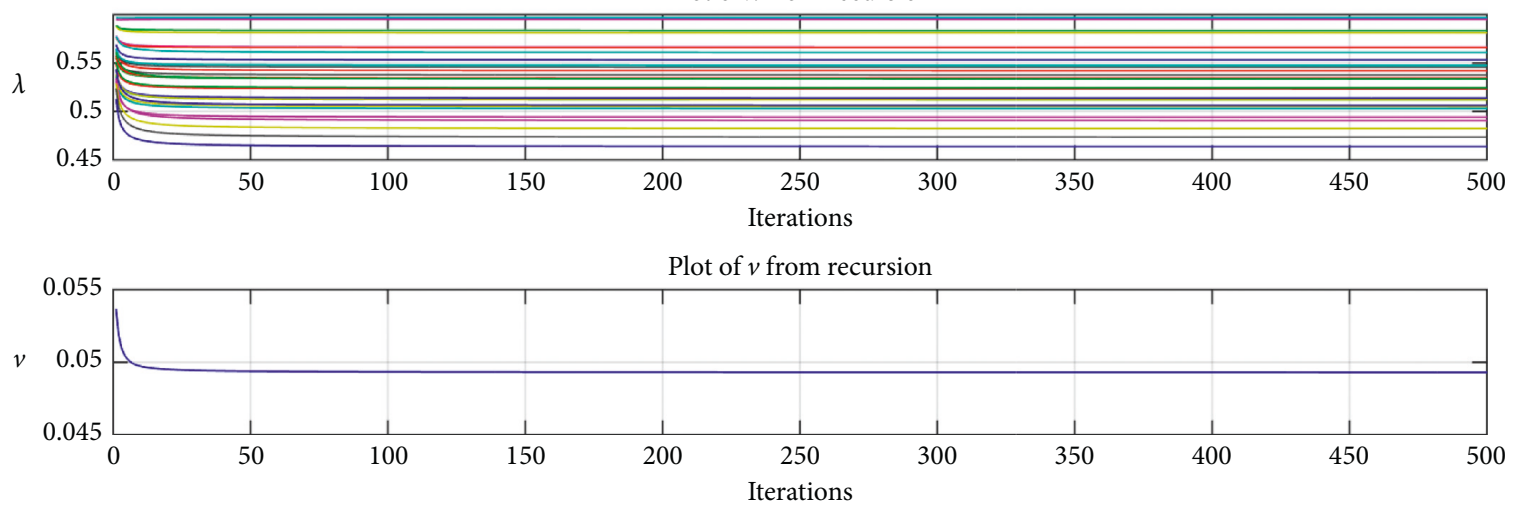

Figure 9: Convergence of $\eta, \lambda$, and $v$ with increasing iterations.

\section{Conclusion}

This paper optimized the power allocation and user association to achieving fairness among different users in underlay CR-based HetNets. Specifically, we adopted a maxmin-based fairness framework under various practical constraints. Problem was first transformed into a standard maximization optimization, and then dual decomposition is used to solve the integer programming problem. The dual problem was solved through the subgradient method. The power allocation at each node is obtained from the convex optimization techniques for the known power allocation at all other nodes. The results are compared with the suboptimal scenarios when the fixed channel allocation is assumed for the optimized/nonoptimized power allocation. The results showed that the proposed scheme outperforms all other candidates, and the spectral efficiency has been improved due to the simultaneous transmission with the primary network.

\section{Appendix}

The Lagrangian $L$ associated with the optimization problem P5 is

$$
\min _{P_{a, c, b}}\left(-\lambda_{a} \log _{2}\left(1+\operatorname{SINR}_{a, c, b}\right)+\eta_{b} P_{a, c, b}+v P_{a, c, b} h_{a, c, b}\right) .
$$

In the above expression, we have converted our problem from a constrained problem to an unconstrained problem.

$$
\begin{aligned}
& \min _{P_{a, c b \geq 0}}\left(-\lambda_{a} \log _{2}\left(1+\frac{P_{a, c, b} g_{a, c, b}}{\sum_{a=1}^{A} \sum_{c=1}^{C} \sum_{b=1}^{B} P_{a, c, b}^{\prime} f_{a, c, b}+\sigma_{a}^{2}}\right)\right. \\
& \left.+\eta_{b} P_{a, c, b}+v P_{a, c, b} h_{a, c, b}\right) .
\end{aligned}
$$

By substituting the value of SINR, we get 


$$
\begin{aligned}
= & \frac{\partial}{\partial P_{a, c, b}}\left(-\lambda_{a} \log _{2}\left(1+\frac{P_{a, c, b} g_{a, c, b}}{\sum_{a=1}^{A} \sum_{c=1}^{C} \sum_{b=1}^{B} P_{a, c, b}^{\prime} f_{a, c, b}+\sigma_{a}^{2}}\right)\right. \\
& \left.+\eta_{b} P_{a, c, b}+v P_{a, c, b} h_{a, c, b}\right) .
\end{aligned}
$$

Applying the KKT conditions for optimality, and by solving the first derivative test, we obtain

$$
\begin{aligned}
= & -\lambda_{a}\left(\frac{1}{1+\left(P_{a, c, b}+g_{a, c, b} / \sum_{a=1}^{A} \sum_{c=1}^{C} \sum_{b=1}^{B} P_{a, c, b}^{\prime} f_{a, c, b}+\sigma_{a}^{2}\right)}\right) \\
& \times \frac{\partial}{\partial P_{a, c, b}}\left(1+\frac{P_{a, c, b} g_{a, c, b}}{\sum_{a=1}^{A} \sum_{c=1}^{C} \sum_{b=1}^{B} P_{a, c, b}^{\prime} f_{a, c, b}+\sigma_{a}^{2}}\right)+\eta_{b}+v h_{a, c, b} .
\end{aligned}
$$

Now, solving the partial derivative as

$$
\begin{aligned}
= & \eta_{b}+v h_{a, c, b}-\lambda_{a} \\
& \times\left(\frac{\sum_{a=1}^{A} \sum_{c=1}^{C} \sum_{b=1}^{B} P_{a, c, b}^{\prime} f_{a, c, b}+\sigma_{a}^{2}}{\left(\sum_{a=1}^{A} \sum_{c=1}^{C} \sum_{b=1}^{B} P_{a, c, b}^{\prime} f_{a, c, b}+\sigma_{a}^{2}\right)+P_{a, c, b} g_{a, c, b}}\right) \\
& \times\left(\frac{g_{a, c, b} \sum_{a=1}^{A} \sum_{c=1}^{C} \sum_{b=1}^{B} P_{a, c, b}^{\prime} f_{a, c, b}+\sigma_{a}^{2}}{\left(\sum_{a=1}^{A} \sum_{c=1}^{C} \sum_{b=1}^{B} P_{a, c, b}^{\prime} f_{a, c, b}+\sigma_{a}^{2}\right)^{2}}\right) .
\end{aligned}
$$

Taking L.C.M. of previous step and finding the partial derivative of internal function, we have

$$
\begin{aligned}
= & -\lambda_{a}\left(\frac{g_{a, c, b}\left(\sum_{a=1}^{A} \sum_{c=1}^{C} \sum_{b=1}^{B} P_{a, c, b}^{\prime} f_{a, c, b}+\sigma_{a}^{2}\right)^{2}}{\left(\sum_{a=1}^{A} \sum_{c=1}^{C} \sum_{b=1}^{B} P_{a, c, b}^{\prime} f_{a, c, b}+\sigma_{a}^{2}\right)+P_{a, c, b} g_{a, c, b}}\right) \\
& \times\left(\frac{1}{\left(\sum_{a=1}^{A} \sum_{c=1}^{C} \sum_{b=1}^{B} P_{a, c, b}^{\prime} f_{a, c, b}+\sigma_{a}^{2}\right)^{2}}\right)+\eta_{b}+v h_{a, c, b} .
\end{aligned}
$$

Further simplification results in

$$
\begin{aligned}
= & -\lambda_{a}\left(\frac{g_{a, c, b}}{\sum_{a=1}^{A} \sum_{c=1}^{C} \sum_{b=1}^{B} P_{a, c, b}^{\prime} f_{a, c, b}+\sigma_{a}^{2}+P_{a, c, b} g_{a, c, b}}\right) \\
& +\eta_{b}+v h_{a, c, b} .
\end{aligned}
$$

By canceling the common terms and simplifying, we have

$$
\begin{aligned}
-\eta_{b}-v h_{a, c, b}= & -\lambda_{a}\left(\frac{g_{a, c, b}}{\left(\sum_{a=1}^{A} \sum_{c=1}^{C} \sum_{b=1}^{B} P_{a, c, b}^{\prime} f_{a, c, b}+\sigma_{a}^{2}+P_{a, c, b} g_{a, c, b}\right)+P_{a, c, b} g_{a, c, b}}\right) \\
& \cdot \frac{1}{\left(\sum_{a=1}^{A} \sum_{c=1}^{C} \sum_{b=1}^{B} P_{a, c, b}^{\prime} f_{a, c, b}+\sigma_{a}^{2}+P_{a, c, b} g_{a, c, b}\right)+P_{a, c, b} g_{a, c, b}}=\frac{\eta_{b}+v h_{a, c, b}}{\lambda_{a, c, b} g_{a, c, b}} .
\end{aligned}
$$

After some mathematical simplifications, we get

$$
\begin{gathered}
\left(\sum_{a=1}^{A} \sum_{c=1}^{C} \sum_{b=1}^{B} P_{a, c, b}^{\prime} f_{a, c, b}+\sigma_{a}^{2}+P_{a, c, b} g_{a, c, b}\right) \\
+P_{a, c, b} g_{a, c, b}=\frac{\lambda_{a, c, b} g_{a, c, b}}{\eta_{b}+v h_{a, c, b}} .
\end{gathered}
$$

Inverting both sides of the equation as

$$
\begin{aligned}
P_{a, c, b} g_{a, c, b}= & \left(\frac{\lambda_{a, c, b} g_{a, c, b}}{\eta_{b}+v h_{a, c, b}}\right) \\
& -\left(\sum_{a=1}^{A} \sum_{c=1}^{C} \sum_{b=1}^{B} P_{a, c, b}^{\prime} f_{a, c, b}+\sigma_{a}^{2}+P_{a, c, b} g_{a, c, b}\right) .
\end{aligned}
$$

After cross multiplying, we have

$$
P_{a, c, b}=\frac{\lambda_{a, c, b} g_{a, c, b}-\left(\Xi \eta_{b}+v h_{a, c, b}\right)}{\eta_{b}+v h_{a, c, b}},
$$

where $\Xi=\left(\sum_{a=1}^{A} \sum_{c=1}^{C} \sum_{b=1}^{B} P_{a, c, b}^{\prime} f_{a, c, b}+\sigma_{a}^{2}\right)$.

\section{Data Availability}

The data used to support the findings of this study are available from the corresponding author upon request.

\section{Conflicts of Interest}

The authors declare that there are no conflicts of interest regarding the publication of this paper. 


\section{Acknowledgments}

This research was supported by the Chung-Ang University research grant in 2017 and the Basic Science Research Program through the National Research Foundation of Korea funded by the Ministry of Education (NRF2019R1F1A1042599).

\section{References}

[1] F. Jameel, Z. Hamid, F. Jabeen, S. Zeadally, and M. A. Javed, "A survey of device-to-device communications: research issues and challenges," IEEE Communications Surveys \& Tutorials, vol. 20, no. 3, pp. 2133-2168, 2018.

[2] B. Wang, F. Gao, S. Jin, H. Lin, and G. Y. Li, "Spatial- and frequency-wideband effects in millimeter-wave massive MIMO systems," IEEE Transactions on Signal Processing, vol. 66, no. 13, pp. 3393-3406, 2018.

[3] X. Wang, E. Turgut, and M. Cenk Gursoy, "Coverage in downlink heterogeneous mmWave cellular networks with user-centric small cell deployment," 2017, https://arxiv.org/ abs/1707.07035.

[4] Y. Xu, Y. Hu, and G. Li, "Robust rate maximization for heterogeneous wireless networks under channel uncertainties," Sensors, vol. 18, no. 2, p. 639, 2018.

[5] Y. Xu and S. Mao, "User association in massive MIMO HetNets," IEEE Systems Journal, vol. 11, no. 1, pp. 7-19, 2017.

[6] A. Khandekar, N. Bhushan, J. Tingfang, and V. Vanghi, "LTEadvanced: heterogeneous networks," in Proceedings of the 2010 European Wireless Conference (EW), pp. 978-982, Lucca, Italy, April 2010.

[7] F. Jameel, S. Kumar, Z. Chang, T. Hamalainan, and T. Ristaniemi, "Operator revenue analysis for device-to-device communications overlaying cellular network," in Proceedings of the 2018 IEEE Conference on Standards for Communications and Networking (CSCN), pp. 1-6, Paris, France, October 2018.

[8] Y. Sun, W. Xia, S. Zhang, Y. Wu, T. Wang, and Y. Fang, "Energy efficient pico cell range expansion and density joint optimization for heterogeneous networks with eICIC," Sensors, vol. 18, no. 3, p. 762, 2018.

[9] H. Zhang, H. Ji, and Y. Wang, "User association scheme in heterogeneous networks considering multiple real-world policies," in Proceedings of the National Doctoral Academic Forum on Information and Communications Technology 2013, Beijing, China, 2013.

[10] K. Cwalina, S. Ambroziak, P. Rajchowski, J. Sadowski, and J. Stefanski, "A novel bitrate adaptation method for heterogeneous wireless body area networks," Applied Sciences, vol. 8, no. 7, p. 1209, 2018.

[11] D. Fooladivanda and C. Rosenberg, "Joint resource allocation and user association for heterogeneous wireless cellular networks," IEEE Transactions on Wireless Communications, vol. 12, no. 1, pp. 248-257, 2013.

[12] W. U. Khan, Z. Ali, M. Waqas, and G. A. S. Sidhu, "Efficient power allocation with individual QoS guarantees in future small-cell networks," AEU-International Journal of Electronics and Communications, vol. 105, pp. 36-41, 2019.

[13] K. Shen and W. Yu, "Downlink cell association optimization for heterogeneous networks via dual coordinate descent," in Proceedings of the 2013 IEEE International Conference on Acoustics, Speech and Signal Processing, pp. 4779-4783, IEEE, Vancouver, Canada, May 2013.

[14] K. Shen and W. Yu, "Distributed pricing-based user association for downlink heterogeneous cellular networks," IEEE
Journal on Selected Areas in Communications, vol. 32, no. 6, pp. 1100-1113, 2014.

[15] D. Liu, Y. Chen, K. K. Chai, and T. Zhang, "Nash bargaining solution based user association optimization in HetNets," in Proceedings of the 2014 IEEE 11th Consumer Communications and Networking Conference (CCNC), pp. 587-592, IEEE, Las Vegas, NV, USA, January 2014.

[16] P. Xue, P. Gong, J. H. Park, D. Park, and D. K. Kim, “Max-min fairness based radio resource management in fourth generation heterogeneous networks," in Proceedings of the 2009 9th International Symposium on Communications and Information Technology, pp. 208-213, IEEE, Icheon, South Korea, September 2009.

[17] W. U. Khan, F. Jameel, T. Ristaniemi, B. M. Elhalawany, and J. Liu, "Efficient power allocation for multi-cell uplink NOMA network," in Proceedings of the 2019 IEEE 89th Vehicular Technology Conference (VTC2019-Spring), pp. 1-5, IEEE, Kuala Lumpur, Malaysia, April-May 2019.

[18] R. Amin, J. Martin, J. Deaton, L. A. DaSilva, A. Hussien, and A. Eltawil, "Balancing spectral efficiency, energy consumption, and fairness in future heterogeneous wireless systems with reconfigurable devices," IEEE Journal on Selected Areas in Communications, vol. 31, no. 5, pp. 969-980, 2013.

[19] C. Qin, C. Wang, D. Pan, W. Wang, and Y. Zhang, "A cross time slot partial interference alignment scheme in two-cell relay heterogeneous networks," Applied Sciences, vol. 9, no. 4, p. 652, 2019.

[20] T. Jabeen, Z. Ali, W. U. Khan et al., "Joint power allocation and link selection for multi-carrier buffer aided relay network," Electronics, vol. 8, no. 6, p. 686, 2019.

[21] A. Khodmi, S. B. R. Chaouch, N. Agoulmine, and Z. Choukair, "A joint power allocation and user association based on noncooperative game theory in an heterogeneous ultra-dense network," IEEE Access, vol. 7, pp. 111790-111800, 2019.

[22] P. T. Semov, V. Poulkov, A. Mihovska, and R. Prasad, "Increasing throughput and fairness for users in heterogeneous semi coordinated deployments," in Proceedings of the 2014 IEEE Wireless Communications and Networking Conference Workshops (WCNCW), pp. 40-45, IEEE, Istanbul, Turkey, April 2014.

[23] Q. Li, R. Q. Hu, Y. Qian, and G. Wu, "A proportional fair radio resource allocation for heterogeneous cellular networks with relays," in Proceedings of the 2012 IEEE Global Communications Conference (GLOBECOM), pp. 5457-5463, IEEE, Anaheim, CA, USA, December 2012.

[24] J. Jiang, W. Wang, M. Peng, and Y. Huang, "Energy efficiency comparison between orthogonal and co-channel resource allocation schemes in distributed antenna systems," in Proceedings of the 2012 IEEE 23rd International Symposium on Personal, Indoor and Mobile Radio Communications(PIMRC), pp. 541-545, IEEE, Sydney, Australia, September 2012.

[25] J. Jiang, M. Peng, K. Zhang, and L. Li, "Energy-efficient resource allocation in heterogeneous network with cross-tier interference constraint," in Proceedings of the 2013 IEEE 24th International Symposium on Personal, Indoor and Mobile Radio Communications (PIMRC Workshops), pp. 168-172, IEEE, London, UK, September 2013.

[26] X. Wang, M. Jia, Q. Guo, I. W.-H. Ho, and J. Wu, "Joint power, original bandwidth, and detected hole bandwidth allocation for multi-homing heterogeneous networks based on cognitive radio," IEEE Transactions on Vehicular Technology, vol. 68, no. 3, pp. 2777-2790, 2019. 
[27] X. Liu, Y. Zhang, Y. Li, Z. Zhang, and K. Long, "A survey of cognitive radio technologies and their optimization approaches," in Proceedings of the 2013 8th International Conference on Communications and Networking in China (CHINACOM), pp. 973-978, IEEE, Guilin, China, August 2013.

[28] H. O. Kpojime and G. A. Safdar, "Interference mitigation in cognitive-radio-based femtocells," IEEE Communications Surveys \& Tutorials, vol. 17, no. 3, pp. 1511-1534, 2015.

[29] Y. Wang, W. Xu, K. Yang, and J. Lin, "Optimal energy-efficient power allocation for OFDM-based cognitive radio networks," IEEE Communications Letters, vol. 16, no. 9, pp. 1420-1423, 2012.

[30] L. Zhang, T. Jiang, and K. Luo, "Dynamic spectrum allocation for the downlink of OFDMA-based hybrid-access cognitive femtocell networks," IEEE Transactions on Vehicular Technology, vol. 65, no. 3, pp. 1772-1781, 2016.

[31] R. Liu, M. Sheng, and W. Wu, "Energy-efficient resource allocation for heterogeneous wireless network with multihomed user equipments," IEEE Access, vol. 6, pp. 1459114601, 2018.

[32] Y. Xu, Y. Hu, Q. Chen, R. Chai, and G. Li, "Distributed resource allocation for cognitive HetNets with cross-tier interference constraint," in Proceedings of the 2017 IEEE Wireless Communications and Networking Conference (WCNC), pp. 1-6, San Francisco, CA, USA, March 2017.

[33] W. Yu and R. Lui, "Dual methods for nonconvex spectrum optimization of multicarrier systems," IEEE Transactions on Communications, vol. 54, no. 7, pp. 1310-1322, 2006. 




\section{Enfincering}
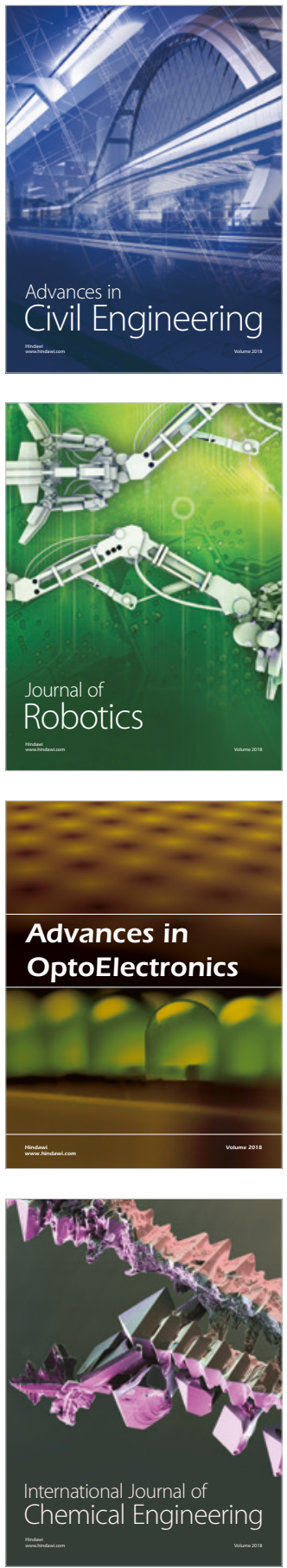



\section{Rotating \\ Machinery}

The Scientific World Journal

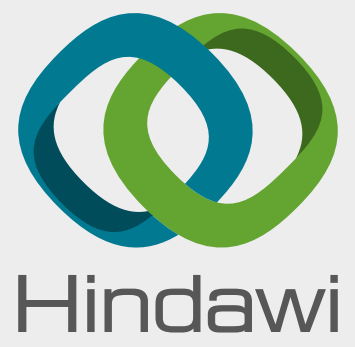

Submit your manuscripts at

www.hindawi.com
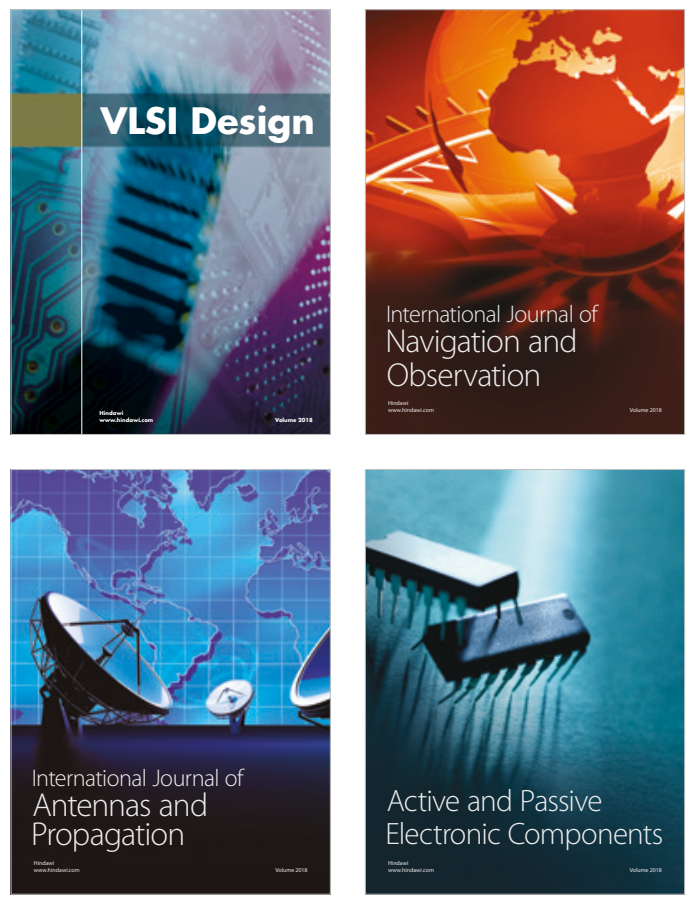
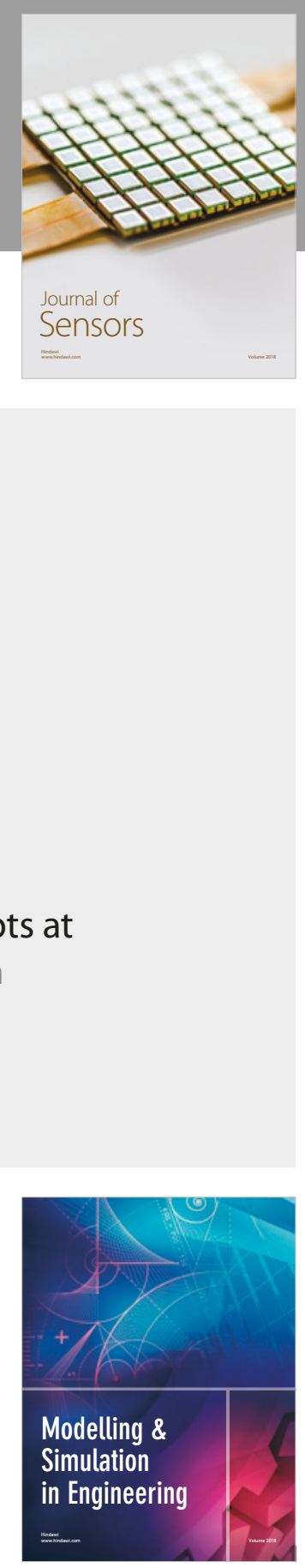

\section{Advances \\ Multimedia}
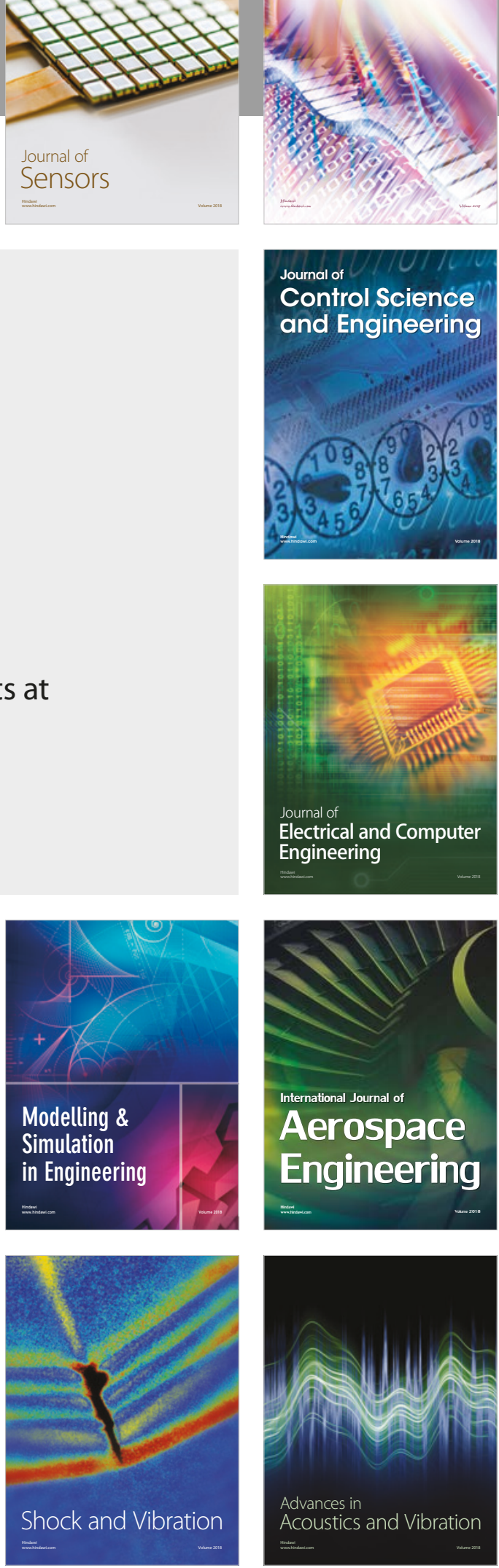